\title{
Inner functions as improving multipliers and zero sets of Besov-type spaces
}

\section{Zengjian Lou* and Ruishen Qian}

"Correspondence: zjlou@stu.edu.cn Department of Mathematics, Shantou University, Guangdong, Shantou 515063, P.R. China

\begin{abstract}
Assume $X$ and $Y$ are two spaces of analytic functions in the unit disk $\mathbb{D}$ with $X \subseteq Y$. Let $\theta$ be an inner function. If every function $f \in X$ satisfying $f \theta \in Y$ must actually satisfy $f \theta \in X$, then $\theta$ is said to be $(X, Y)$-improving. In this paper, we characterize the inner functions in the Möbius invariant Besov-type spaces $F(p, p-2, s)$ as improving multipliers for $p>1$ and $0<s<1$. Our result generalizes Peláez's result on $Q_{s}$ spaces $(0<s<1)$ (Peláez in J. Funct. Anal. 255:1403-1418, 2008).
\end{abstract}

MSC: 30D45; 30D50

Keywords: inner functions; improving; zero set; Carleson-Newman sequence; Besov-type space

\section{Introduction}

We denote the unit disk $\{z \in \mathbb{C}:|z|<1\}$ by $\mathbb{D}$ and its boundary $\{z \in \mathbb{C}:|z|=1\}$ by $\partial \mathbb{D}$. Let $H(\mathbb{D})$ be the space of all analytic functions in $\mathbb{D}$. An analytic function in the unit disc $\mathbb{D}$ is called an inner function if it is bounded and the modulus equals 1 almost everywhere on the boundary $\partial \mathbb{D}$.

It is well known that every inner function has a factorization $e^{i \gamma} B(z) S(z)$, where $\gamma \in \mathbb{R}$, $B(z)$ is a Blaschke product and $S(z)$ is a singular inner function, that is,

$$
B(z)=\prod_{k=1}^{\infty} \frac{\left|a_{k}\right|}{a_{k}} \frac{a_{k}-z}{1-\bar{a}_{k} z}
$$

and

$$
S(z)=\exp \left\{-\int_{0}^{2 \pi} \frac{e^{i t}+z}{e^{i t}-z} d \mu(t)\right\}
$$

where $\left\{a_{k}\right\}_{k=1}^{\infty}$ is a sequence of points in $\mathbb{D}$ which satisfies the Blaschke condition

$$
\sum_{k=1}^{\infty}\left(1-\left|a_{k}\right|\right)<\infty
$$

and $\mu$ is a finite positive Borel measure in $[0,2 \pi)$, which is singular with respect to Lebesgue measure. Let $\sigma(\theta)$ denote the singular set or boundary spectrum of inner function $\theta$. From [1] we know that $\sigma(\theta) \subseteq \partial \mathbb{D}$ is the smallest closed set such that $\theta$ is analytic

\section{Springer}

(2014 Lou and Qian; licensee Springer. This is an Open Access article distributed under the terms of the Creative Commons Attribution License (http://creativecommons.org/licenses/by/2.0), which permits unrestricted use, distribution, and reproduction in any medium, provided the original work is properly cited. 
across $\partial \mathbb{D} \backslash \sigma(\theta)$, and $\sigma(\theta)$ consists of the accumulation points of zeros of $\theta$ and the closed support of the associated singular measure. See [2-6] for more information on inner functions.

Since a nontrivial inner function $\theta$ is extremely oscillatory near $\sigma(\theta)$, the same should happen to the product $f \theta$, where $f \in H(\mathbb{D})$ is smooth in some sense on $\partial \mathbb{D}$. But sometimes the product $f \theta$ inherits the nice properties of $f$, and it is possible that $f \theta$ has an added smoothness. In order to analyze this phenomenon, Dyakonov introduced the following notion in [7].

Suppose $X$ and $Y$ are two classes of analytic functions on $\mathbb{D}$, and $X \subseteq Y$. Let $\theta$ be an inner function, $\theta$ is said to be $(X, Y)$-improving, if every function $f \in X$ satisfying $f \theta \in Y$ must actually satisfy $f \theta \in X$.

In this paper, we study the inner functions in the Möbius invariant Besov-type spaces $F(p, p-2, s)$ as improving multipliers.

Let $0<p<\infty,-2<q<\infty, s \geq 0$, the $F(p, q, s)$ space is the set of $f \in H(\mathbb{D})[8]$ such that

$$
\|f\|_{F(p, q, s)}=|f(0)|+\left(\sup _{a \in \mathbb{D}} \int_{\mathbb{D}}\left|f^{\prime}(z)\right|^{p}\left(1-|z|^{2}\right)^{q} g(z, a)^{s} d A(z)\right)^{\frac{1}{p}}<\infty
$$

where $g$ denotes the Green function given by

$$
g(z, a)=\log \frac{1}{\left|\varphi_{a}(z)\right|}, \quad z, a \in \mathbb{D}, z \neq a,
$$

$\varphi_{a}(z)=\frac{a-z}{1-\bar{a} z}, d A(z)=\frac{1}{\pi} d x d y$. It is easy to check that $F(p, p-2, s)$ is a Möbius invariant Besov-type space. From [8], when $0<s<1, F(2,0, s)=\mathcal{Q}_{s}$, for more information on $\mathcal{Q}_{s}$ spaces, we refer to [9] and [10]. When $s=1, F(2,0, s)=B M O A$, the space of analytic functions in the Hardy space $H^{1}(\mathbb{D})$ whose boundary functions have bounded mean oscillation. When $s>1, F(2,0, s)=\mathcal{B}$, the Bloch space (see [11]).

The following result was proved by Peláez in [12, Theorem 1].

Theorem A Suppose that $0<s<1$ and $\theta$ is an inner function. Then the following conditions are equivalent:

(1) $\theta \in \mathcal{Q}_{s}$;

(2) $\theta$ is $\left(\mathcal{Q}_{s}, B M O A\right)$-improving;

(3) $\theta$ is $\left(\mathcal{Q}_{s}, \mathcal{B}\right)$-improving.

In this paper, we extend Theorem A from $\mathcal{Q}_{s}$ spaces to a more general space $F(p, p-2, s)$, $0<s<1$.

Theorem 1 Let $1<p<\infty, 0<s<1$. Suppose that $\theta$ is an inner function. Then the following conditions are equivalent:

(1) $\theta \in F(p, p-2, s)$;

(2) $\theta$ is $(F(p, p-2, s), B M O A)$-improving;

(3) $\theta$ is $(F(p, p-2, s), \mathcal{B})$-improving.

The proof of Theorem 1 is gave in Section 3. Theorem 1 can be farther generalized to a more general space $A B_{p}^{t} \cap F(p, p-2, s)$, where $A B_{p}^{t}$ space is the set of functions $f \in H(\mathbb{D})$ 
such that (see [13])

$$
\|f\|_{A B_{p}^{t}}=|f(0)|+\left(\int_{\mathbb{D}}\left|f^{\prime}(z)\right|^{p}(1-|z|)^{(1-t) p-1} d A(z)\right)^{\frac{1}{p}}<\infty .
$$

Corollary 1 Let $1<p<\infty, 0<t p<1$ and $0<s<1$. Suppose $\theta$ is an inner function. Then the following conditions are equivalent:

(1) $\theta \in A B_{p}^{t} \cap F(p, p-2, s)$;

(2) $\theta$ is $\left(A B_{p}^{t} \cap F(p, p-2, s), B M O A\right)$-improving;

(3) $\theta$ is $\left(A B_{p}^{t} \cap F(p, p-2, s), \mathcal{B}\right)$-improving.

Notice the fact that $F(p, p-2, s) \subset A B_{p}^{\frac{1-s}{p}}$. The proof of Corollary 1 is similar to that of Theorem 1 and thus is omitted.

Remark 1 From Theorem 1, we know that any inner function $\theta \in F(p, p-2, s)$ is $(F(p, p-$ $2, s), F(p, p-2, t))$-improving, when $p>1$ and $0<s<t<1$. Conversely, by using the following results (Theorem 2 and Proposition 1 ) on zero sets of Besov-type spaces, we will prove that there exists an inner function $\theta$ which is $(F(p, p-2, s), F(p, p-2, t))$-improving, but $\theta$ does not belong to $F(p, p-2, s)$.

In order to state Theorem 2, we need a few notions.

A Blaschke product $B$ with sequence of zeros $\left\{a_{k}\right\}_{k=1}^{\infty}$ is called interpolating if there exists a positive constant $\delta$ such that

$$
\prod_{j \neq k} \varrho\left(a_{j}, a_{k}\right) \geq \delta, \quad k=1,2, \ldots
$$

Here $\varrho\left(a_{j}, a_{k}\right)=\left|\varphi_{a_{j}}\left(a_{k}\right)\right|$ denotes the pseudo-hyperbolic metric in $\mathbb{D}$. We also say that $\left\{a_{k}\right\}_{k=1}^{\infty}$ is an interpolating sequence or an uniformly separated sequence. A finite union of interpolating sequences is usually called a Carleson-Newman sequence. Similarly, a Carleson-Newman Blaschke product is a finite product of interpolating Blaschke products.

We recall that a function $g \in H(\mathbb{D})$ is called an outer function if $\log |g| \in L^{1}(\partial \mathbb{D})$ and

$$
g(z)=\eta \exp \left(\frac{1}{2 \pi} \int_{0}^{2 \pi} \log \left|g\left(e^{i t}\right)\right| \frac{e^{i t}+z}{e^{i t}-z} d t\right)
$$

where $\eta \in \partial \mathbb{D}$.

We say that $Z=\left\{z_{n}\right\} \subset \mathbb{D}$ is a zero set of an analytic function space $X$ defined on $\mathbb{D}$ if there is a $f \in X$ that vanishes on $Z$ and nowhere else. Although the study of zero sets for analytic function spaces is a difficult problem, there are some excellent papers related to this question. We may refer to Carleson [14, 15], Caughran [16], Shapiro and Shields [17], Taylor and Williams [18]. Recently, Pau and Peláez obtained a theorem on zero sets of Dirichlet spaces $\mathcal{D}_{s}=A B_{2}^{\frac{1-s}{2}}$ in [19, Theorem 1]. In the next theorem, we characterize the zero sets of $A B_{p}^{t}$ spaces which generalizes the Pau and Peláez's result in [19]. We characterize the Carleson-Newman sequences that are zero sets in $A B_{p}^{t}$ spaces. 
Theorem 2 Suppose $p>1,0<t<1,0<p t<1$ and $\left\{a_{k}\right\}_{k=1}^{\infty}$ is a Carleson-Newman sequence. Then the following conditions are equivalent:

(1) $\left\{a_{k}\right\}_{k=1}^{\infty}$ is an $A B_{p}^{t}$-zero set;

(2) there exists an outer function $g \in A B_{p}^{t}$ such that

$$
\sum_{k=1}^{\infty}\left|g\left(a_{k}\right)\right|^{p}\left(1-\left|a_{k}\right|^{2}\right)^{1-t p}<\infty
$$

(3) there exists an outer function $g \in A B_{p}^{t}$ such that

$$
\sum_{k=1}^{\infty}\left(1-\left|a_{k}\right|^{2}\right)^{2-t p} \int_{\partial \mathbb{D}} \frac{\left|g\left(e^{i t}\right)\right|^{p}}{\left|e^{i t}-a_{k}\right|^{2}} d t<\infty
$$

(4) there exists an outer function $g \in A B_{p}^{t}$ such that

$$
\sum_{k=1}^{\infty}\left(1-\left|a_{k}\right|^{2}\right)^{1-t p+p}\left(\int_{\partial \mathbb{D}} \frac{\left|g\left(e^{i t}\right)\right|}{\left|e^{i t}-a_{k}\right|^{2}} d t\right)^{p}<\infty .
$$

Using Theorem 2, we can deduce the following result.

Proposition 1 Suppose $p>1,0<t<1,0<p t<1$. There exists a Carleson-Newman sequence $\left\{a_{k}\right\}_{k=1}^{\infty}$ which is not an $A B_{p}^{t}$-zero set and with 1 as unique accumulation point.

The proof of Proposition 1 is similar to that of Theorem 2 of [19] and is omitted here. Applying Proposition 1, we can prove the following result whose proof (as well as the proof of Theorem 2) is given in Section 4.

Corollary 2 Let $p>1$ and $0<s<t<1$. Then there exists an inner function $\theta$ which is $(F(p, p-2, s), F(p, p-2, t))$-improving, but $\theta$ does not belong to $F(p, p-2, s)$.

Throughout this paper, for two functions $f$ and $g, f \asymp g$ means that $g \lesssim f \lesssim g$, that is, there are positive constants $C_{1}$ and $C_{2}$ depending only on the index $p, s, t, \ldots$, such that $C_{1} g \leq f \leq C_{2} g$.

\section{Preliminaries}

To prove Theorem 1 we need some auxiliary results. Lemmas 2.1 and 2.2 should be known to some experts, but we cannot find a reference. For the completeness of the paper, we give proofs below.

Lemma 2.1 Let $0<p<\infty$ and $s \geq 0$. Then $f \in F(p, p-2, s)$ if and only if

$$
\sup _{a \in \mathbb{D}}\left\|f \circ \varphi_{a}-f(a)\right\|_{A B_{p}^{\frac{1-s}{p}}}<\infty
$$


Proof From Theorem 2.4 of [8] and making the change of variables $w=\varphi_{a}(z)$, we have

$$
\begin{aligned}
\|f-f(0)\|_{F(p, p-2, s)}^{p} & =\sup _{a \in \mathbb{D}} \int_{\mathbb{D}}\left|f^{\prime}(w)\right|^{p}\left(1-|w|^{2}\right)^{p-2} g^{s}(w, a) d A(w) \\
& \asymp \sup _{a \in \mathbb{D}} \int_{\mathbb{D}}\left|f^{\prime}(w)\right|^{p}\left(1-|w|^{2}\right)^{p-2}\left(1-\left|\varphi_{a}(w)\right|^{2}\right)^{s} d A(w) \\
& =\sup _{a \in \mathbb{D}} \int_{\mathbb{D}}\left|\left(f \circ \varphi_{a}\right)^{\prime}(z)\right|^{p}\left(1-|z|^{2}\right)^{s+p-2} d A(z) \\
& \asymp \sup _{a \in \mathbb{D}}\left\|f \circ \varphi_{a}-f(a)\right\|_{A B_{p}^{p}}^{p} .
\end{aligned}
$$

For $0<p<\infty, H^{p}$ denotes the Hardy space of $f \in H(\mathbb{D})$ with

$$
\|f\|_{H^{p}}^{p}=\sup _{0<r<1} \frac{1}{2 \pi} \int_{0}^{2 \pi}\left|f\left(r e^{i \theta}\right)\right|^{p} d \theta<\infty
$$

From [20], we know that

$$
\|f\|_{B M O A}=|f(0)|+\sup _{a \in \mathbb{D}}\left\|f \circ \varphi_{a}-f(a)\right\|_{H^{p}}<\infty
$$

can be defined as a norm of $B M O A$ space.

Lemma 2.2 Let $1<p<\infty, 0<s<1$. Then $F(p, p-2, s) \subseteq B M O A$.

Proof From Lemma 2.4 of [21], we know $A B_{p}^{\frac{1-s}{p}} \subset H^{p}$ and

$$
\|f\|_{H^{p}} \leq\|f\|_{A B_{p}} \frac{1-s}{p} .
$$

Let $f \in F(p, p-2, s)$. By Lemma 2.1, we get

$$
\begin{aligned}
\|f-f(0)\|_{B M O A} & =\sup _{a \in \mathbb{D}}\left\|f \circ \varphi_{a}-f(a)\right\|_{H^{p}} \\
& \leq \sup _{a \in \mathbb{D}}\left\|f \circ \varphi_{a}-f(a)\right\|_{A B_{p}^{p}} \frac{1-s}{p} \\
& \asymp\|f-f(0)\|_{F(p, p-2, s)}<\infty .
\end{aligned}
$$

That is, $f \in B M O A$.

The following three lemmas will be used in the proof of Lemma 2.6. Their proofs can be found in [22, Corollary 2.4], [23, Lemma 2.5] or [24, Lemma 1] and [25, Lemma 2.1], respectively.

Lemma 2.3 Let $\theta$ be an inner function and let $1 \leq p<\infty,-2<q<\infty$ and $0<s<\infty$ such that $0<q+s+1<p$. Then, for any $f \in H(\mathbb{D})$ and $a \in \mathbb{D}$,

$$
\begin{gathered}
\int_{\mathbb{D}}|f(z)|^{p}\left(1-|\theta(z)|^{2}\right)^{p}\left(1-|z|^{2}\right)^{q-p}\left(1-\left|\varphi_{a}(z)\right|^{2}\right)^{s} d A(z) \\
\asymp \int_{\mathbb{D}}|f(z)|^{p}\left|\theta^{\prime}(z)\right|^{p}\left(1-|z|^{2}\right)^{q}\left(1-\left|\varphi_{a}(z)\right|^{2}\right)^{s} d A(z) .
\end{gathered}
$$


Lemma 2.4 Let $s>-1, r, t>0$, and $t<s+2<r$. Then

$$
\int_{\mathbb{D}} \frac{\left(1-|w|^{2}\right)^{s}}{|1-\bar{w} z|^{r}|1-\bar{w} \zeta|^{t}} d A(w) \lesssim \frac{\left(1-|z|^{2}\right)^{2+s-r}}{|1-\bar{\zeta} z|^{t}}, \quad z, \zeta \in \mathbb{D}
$$

Lemma 2.5 Let $1<p<\infty$, and $a>-1, b \geq 0$ with $b<2+a, w \in \mathbb{D}$. If $\in H(\mathbb{D})$, then

$$
\int_{\mathbb{D}}|f(z)-f(0)|^{p} \frac{\left(1-|z|^{2}\right)^{a}}{|1-\bar{w} z|^{b}} d A(z) \lesssim \int_{\mathbb{D}}\left(1-|z|^{2}\right)^{p}\left|f^{\prime}(z)\right|^{p} \frac{\left(1-|z|^{2}\right)^{a}}{|1-\bar{w} z|^{b}} d A(z)
$$

Lemma 2.6 plays an important role in the proof of Theorem 1, which generalizes Theorem 6 of [12], with a different proof motivated by [21] and [19].

Lemma 2.6 Let $1<p<\infty, 0<s<1, f \in F(p, p-2, s)$ and $B$ be a Carleson-Newman Blaschke product with a sequence of zeros $\left\{a_{k}\right\}_{k=1}^{\infty}$. Then $f B \in F(p, p-2, s)$ if and only if

$$
\sup _{a \in \mathbb{D}} \sum_{k=1}^{\infty}\left|f\left(a_{k}\right)\right|^{p}\left(1-\left|\varphi_{a}\left(a_{k}\right)\right|^{2}\right)^{s}<\infty
$$

Proof Necessity. If $f B \in F(p, p-2, s)$, then it is easy to deduce that

$$
\sup _{a \in \mathbb{D}} \int_{\mathbb{D}}|f(z)|^{p}\left|B^{\prime}(z)\right|^{p}\left(1-|z|^{2}\right)^{p-2}\left(1-\left|\varphi_{a}(z)\right|^{2}\right)^{s} d A(z)<\infty
$$

We will prove that

$$
\begin{aligned}
& \sup _{a \in \mathbb{D}} \sum_{k=1}^{\infty}\left|f\left(a_{k}\right)\right|^{p}\left(1-\left|\varphi_{a}\left(a_{k}\right)\right|^{2}\right)^{s} \\
& \quad \lesssim \sup _{a \in \mathbb{D}} \int_{\mathbb{D}}|f(z)|^{p}\left|B^{\prime}(z)\right|^{p}\left(1-|z|^{2}\right)^{p-2}\left(1-\left|\varphi_{a}(z)\right|^{2}\right)^{s} d A(z) .
\end{aligned}
$$

From the sub-mean-value property of $|f|^{p}$ and the estimate

$$
1-\left|a_{k}\right|^{2} \asymp 1-|z|^{2} \asymp\left|1-\bar{a}_{k} z\right|, \quad z \in E\left(a_{k}, r\right),
$$

for $r>0$ (see [26, p.69]). Here and afterwards

$$
E\left(a_{k}, r\right)=\left\{z:\left|\varphi_{a_{k}}(z)\right|<r\right\} .
$$

We have

$$
\begin{aligned}
& \sum_{k=1}^{\infty}\left|f\left(a_{k}\right)\right|^{p}\left(1-\left|\varphi_{a}\left(a_{k}\right)\right|^{2}\right)^{s} \\
& \quad \lesssim \sum_{k=1}^{\infty}\left(1-\left|\varphi_{a}\left(a_{k}\right)\right|^{2}\right)^{s} \int_{E\left(a_{k}, r\right)} \frac{|f(z)|^{p}}{\left|1-\bar{a}_{k} z\right|^{2}} d A(z) \\
& \quad \asymp \sum_{k=1}^{\infty} \int_{E\left(a_{k}, r\right)}|f(z)|^{p} \frac{\left(1-\left|a_{k}\right|^{2}\right)^{p}\left(1-|z|^{2}\right)^{p}}{\left|1-\bar{a}_{k} z\right|^{2 p+2}}\left(1-\left|\varphi_{a}(z)\right|^{2}\right)^{s} d A(z)
\end{aligned}
$$




$$
\begin{aligned}
& \asymp \sum_{k=1}^{\infty}\left(1-\left|a_{k}\right|^{2}\right)^{p} \int_{E\left(a_{k}, r\right)}|f(z)|^{p} \frac{\left(1-|z|^{2}\right)^{p-2}}{\left|1-\bar{a}_{k} z\right|^{2 p}}\left(1-\left|\varphi_{a}(z)\right|^{2}\right)^{s} d A(z) \\
& \lesssim \sum_{k=1}^{\infty}\left(1-\left|a_{k}\right|^{2}\right)^{p} \int_{\mathbb{D}}|f(z)|^{p} \frac{\left(1-|z|^{2}\right)^{p-2}}{\left|1-\bar{a}_{k} z\right|^{2 p}}\left(1-\left|\varphi_{a}(z)\right|^{2}\right)^{s} d A(z) \\
& =\int_{\mathbb{D}}|f(z)|^{p} \sum_{k=1}^{\infty}\left(1-\left|\varphi_{a_{k}}(z)\right|^{2}\right)^{p}\left(1-|z|^{2}\right)^{-2}\left(1-\left|\varphi_{a}(z)\right|^{2}\right)^{s} d A(z) \\
& \lesssim \int_{\mathbb{D}}|f(z)|^{p}\left(\sum_{k=1}^{\infty}\left(1-\left|\varphi_{a_{k}}(z)\right|^{2}\right)\right)^{p}\left(1-|z|^{2}\right)^{-2}\left(1-\left|\varphi_{a}(z)\right|^{2}\right)^{s} d A(z) .
\end{aligned}
$$

Since $B$ is a Carleson-Newman Blaschke product with a sequence of zeros $\left\{a_{k}\right\}_{k=1}^{\infty}$, we have

$$
\sup _{z \in \mathbb{D}} \sum_{k=1}^{\infty}\left(1-\left|\varphi_{z}\left(a_{k}\right)\right|^{2}\right) \lesssim 1 .
$$

Note that

$$
1-r^{2} \leq-2 \log r, \quad 0<r \leq 1
$$

we have

$$
2 \log |B(z)|=\sum_{n=1}^{\infty} \log \left|\varphi_{a_{k}}(z)\right|^{2} \leq-\sum_{k=1}^{\infty}\left(1-\left|\varphi_{a_{k}}(z)\right|^{2}\right) .
$$

Since the function $\frac{\left(1-e^{-x}\right)}{x}$ is decreasing in $(0, \infty)$, we get

$$
\begin{aligned}
1-|B(z)|^{2} & \geq \sum_{k=1}^{\infty}\left(1-\left|\varphi_{a_{k}}(z)\right|^{2}\right) \frac{1-\exp \left(-\sup _{z \in \mathbb{D}} \sum_{k=1}^{\infty}\left(1-\left|\varphi_{a_{k}}(z)\right|^{2}\right)\right)}{\sup _{z \in \mathbb{D}} \sum_{k=1}^{\infty}\left(1-\left|\varphi_{a_{k}}(z)\right|^{2}\right)} \\
& \asymp \sum_{k=1}^{\infty}\left(1-\left|\varphi_{a_{k}}(z)\right|^{2}\right) .
\end{aligned}
$$

Combining this with (1) and using Lemma 2.3 yield

$$
\begin{aligned}
& \sum_{k=1}^{\infty}\left|f\left(a_{k}\right)\right|^{p}\left(1-\left|\varphi_{a}\left(a_{k}\right)\right|^{2}\right)^{s} \\
& \quad \lesssim \int_{\mathbb{D}}|f(z)|^{p}\left|B^{\prime}(z)\right|^{p}\left(1-|z|^{2}\right)^{p-2}\left(1-\left|\varphi_{a}(z)\right|^{2}\right)^{s} d A(z)<\infty .
\end{aligned}
$$

Sufficiency. Applying the $p$-triangle inequality gives

$$
\begin{aligned}
& \int_{\mathbb{D}}\left|(f(z) B(z))^{\prime}\right|^{p}\left(1-|z|^{2}\right)^{p-2}\left(1-\left|\varphi_{a}(z)\right|^{2}\right)^{s} d A(z) \\
& \lesssim \int_{\mathbb{D}}\left|f^{\prime}(z)\right|^{p}|B(z)|^{p}\left(1-|z|^{2}\right)^{p-2}\left(1-\left|\varphi_{a}(z)\right|^{2}\right)^{s} d A(z) \\
& \quad+\int_{\mathbb{D}}|f(z)|^{p}\left|B^{\prime}(z)\right|^{p}\left(1-|z|^{2}\right)^{p-2}\left(1-\left|\varphi_{a}(z)\right|^{2}\right)^{s} d A(z) \\
& =: I_{1}+I_{2} .
\end{aligned}
$$


Since $f \in F(p, p-2, s), B \in H^{\infty}$, by Theorem 2.4 of [8], we have

$$
\begin{aligned}
I_{1} & \lesssim\|B\|_{H^{\infty}}^{p} \int_{\mathbb{D}}\left|f^{\prime}(z)\right|^{p}\left(1-|z|^{2}\right)^{p-2}\left(1-\left|\varphi_{a}(z)\right|^{2}\right)^{s} d A(z) \\
& \lesssim\|f\|_{F(p, p-2, s)}^{p} .
\end{aligned}
$$

We now estimate $I_{2}$. Using the fact that $\left(1-|z|^{2}\right)\left|B^{\prime}(z)\right| \leq 1$ and

$$
\left|B^{\prime}(z)\right| \leq \sum_{k=1}^{\infty} \frac{1-\left|a_{k}\right|^{2}}{\left|1-\bar{a}_{k} z\right|^{2}},
$$

and employing the $p$-triangle inequality again, we have

$$
\begin{aligned}
I_{2} & =\int_{\mathbb{D}}|f(z)|^{p}\left|B^{\prime}(z)\right|^{p}\left(1-|z|^{2}\right)^{p-2}\left(1-\left|\varphi_{a}(z)\right|^{2}\right)^{s} d A(z) \\
& \lesssim \int_{\mathbb{D}}|f(z)|^{p}\left|B^{\prime}(z)\right|\left(1-|z|^{2}\right)^{-1}\left(1-\left|\varphi_{a}(z)\right|^{2}\right)^{s} d A(z) \\
\lesssim & \int_{\mathbb{D}}|f(z)|^{p}\left(\sum_{k=1}^{\infty} \frac{1-\left|a_{k}\right|^{2}}{\left|1-\bar{a}_{k} z\right|^{2}}\right)\left(1-|z|^{2}\right)^{-1}\left(1-\left|\varphi_{a}(z)\right|^{2}\right)^{s} d A(z) \\
= & \sum_{k=1}^{\infty}\left(1-\left|a_{k}\right|^{2}\right) \int_{\mathbb{D}}|f(z)|^{p} \frac{\left(1-|z|^{2}\right)^{-1}}{\left|1-\bar{a}_{k} z\right|^{2}}\left(1-\left|\varphi_{a}(z)\right|^{2}\right)^{s} d A(z) \\
\lesssim & \sum_{k=1}^{\infty}\left(1-\left|a_{k}\right|^{2}\right) \int_{\mathbb{D}}\left|f\left(a_{k}\right)\right|^{p} \frac{\left(1-|z|^{2}\right)^{-1}}{\left|1-\bar{a}_{k} z\right|^{2}}\left(1-\left|\varphi_{a}(z)\right|^{2}\right)^{s} d A(z) \\
& +\sum_{k=1}^{\infty}\left(1-\left|a_{k}\right|^{2}\right) \int_{\mathbb{D}}\left|f(z)-f\left(a_{k}\right)\right|^{p} \frac{\left(1-|z|^{2}\right)^{-1}}{\left|1-\bar{a}_{k} z\right|^{2}}\left(1-\left|\varphi_{a}(z)\right|^{2}\right)^{s} d A(z) \\
= & I_{3}+I_{4} .
\end{aligned}
$$

Applying Lemma 2.4 yields

$$
\begin{aligned}
I_{3} & =\sum_{k=1}^{\infty}\left(1-\left|a_{k}\right|^{2}\right)\left|f\left(a_{k}\right)\right|^{p} \int_{\mathbb{D}} \frac{\left(1-|z|^{2}\right)^{-1}}{\left|1-\bar{a}_{k} z\right|^{2}}\left(1-\left|\varphi_{a}(z)\right|^{2}\right)^{s} d A(z) \\
& \lesssim \sup _{a \in \mathbb{D}} \sum_{k=1}^{\infty}\left|f\left(a_{k}\right)\right|^{p}\left(1-\left|\varphi_{a}\left(a_{k}\right)\right|^{2}\right)^{s}<\infty .
\end{aligned}
$$

Let $\varphi_{\lambda}(w)=e^{i \tau} \varphi_{a}\left(\varphi_{a_{k}}(w)\right), \tau \in[0,2 \pi]$. Making the change of variables $z=\varphi_{a_{k}}(w)$ and using Lemma 2.5, we get

$$
\begin{aligned}
I_{4} & \lesssim \sum_{k=1}^{\infty} \int_{\mathbb{D}}\left|\left(f \circ \varphi_{a_{k}}\right)(w)-\left(f \circ \varphi_{a_{k}}\right)(0)\right|^{p} \frac{\left(1-\left|\varphi_{\lambda}(w)\right|^{2}\right)^{s}}{\left(1-|w|^{2}\right)} d A(w) \\
& =\sum_{k=1}^{\infty}\left(1-|\lambda|^{2}\right)^{s} \int_{\mathbb{D}}\left|\left(f \circ \varphi_{a_{k}}\right)(w)-\left(f \circ \varphi_{a_{k}}\right)(0)\right|^{p} \frac{\left(1-|w|^{2}\right)^{s-1}}{|1-\bar{\lambda} w|^{2 s}} d A(w) \\
& \lesssim \sum_{k=1}^{\infty} \int_{\mathbb{D}}\left|\left(f \circ \varphi_{a_{k}}\right)^{\prime}(w)\right|^{p}\left(1-|w|^{2}\right)^{p-1}\left(1-\left|\varphi_{\lambda}(w)\right|^{2}\right)^{s} d A(w)
\end{aligned}
$$




$$
\begin{aligned}
& =\sum_{k=1}^{\infty} \int_{\mathbb{D}}\left|f^{\prime}(z)\right|^{p}\left(1-|z|^{2}\right)^{p-2}\left(1-\left|\varphi_{a_{k}}(z)\right|^{2}\right)\left(1-\left|\varphi_{a}(z)\right|^{2}\right)^{s} d A(z) \\
& \lesssim \int_{\mathbb{D}}\left|f^{\prime}(z)\right|^{p}\left(1-|z|^{2}\right)^{p-2}\left(1-\left|\varphi_{a}(z)\right|^{2}\right)^{s} d A(z) \lesssim\|f\|_{F(p, p-2, s)}^{p} .
\end{aligned}
$$

Hence, we have $f \theta \in F(p, p-2, s)$.

The following well-known results will also be used in the proof of Theorem 1.

Lemma 2.7 ([22, Theorem 1.4]) Let $0<s<1$. Then an inner function belongs to the Möbius invariant Besov-type space $F(p, p-2, s)$ for all $p>\max \{s, 1-s\}$ if and only if it is the Blaschke product associated with a sequence $\left\{a_{k}\right\}_{k=1}^{\infty}$ which satisfies

$$
\sup _{a \in \mathbb{D}} \sum_{k=1}^{\infty}\left(1-\left|\varphi_{a}\left(a_{k}\right)\right|^{2}\right)^{s}<\infty .
$$

Lemma 2.8 ([27, Lemma 21]) Let $\left\{a_{k}\right\}_{k=1}^{\infty}$ be a sequence in $\mathbb{D}$. Then the measure $d \mu_{a_{k}}=$ $\sum_{k=1}^{\infty}\left(1-\left|a_{k}\right|^{2}\right) \delta_{a_{k}}$ is a Carleson measure, i.e.

$$
\sup _{a \in \mathbb{D}} \sum_{k=1}^{\infty}\left(1-\left|\varphi_{a}\left(a_{k}\right)\right|^{2}\right)<\infty
$$

if and only if $\left\{a_{k}\right\}_{k=1}^{\infty}$ is a finite union of uniformly separated sequences.

If $\theta$ is an inner function, for $0<\epsilon<1$, define the level set of order $\epsilon$ of $\theta$ as

$$
\Omega(\theta, \epsilon)=\{z \in \mathbb{D}:|\theta(z)|<\epsilon\}
$$

Lemma 2.9 ([28, Theorem 1]) If $f \in B M O A$ and $\theta$ is an inner function, then the following conditions are equivalent:

(1) $f \theta \in B M O A$;

(2) $\sup _{z \in \mathbb{D}}|f(z)|^{2}\left(1-|\theta(z)|^{2}\right)<\infty$;

(3) $\sup _{z \in \Omega(\theta, \epsilon)}|f(z)|<\infty$, for every $\epsilon, 0<\epsilon<1$;

(4) $\sup _{z \in \Omega(\theta, \epsilon)}|f(z)|<\infty$, for some $\epsilon, 0<\epsilon<1$.

\section{Proof of Theorem 1}

$(1) \Rightarrow(2)$. For inner functions $\theta \in F(p, p-2, s)$, by Lemma 2.7, $\theta$ is a Blaschke product with zeros $\left\{a_{k}\right\}_{k=1}^{\infty}$, and

$$
\sup _{a \in \mathbb{D}} \sum_{k=1}^{\infty}\left(1-\left|\varphi_{a}\left(a_{k}\right)\right|^{2}\right)^{s}<\infty,
$$

which implies that

$$
\sup _{a \in \mathbb{D}} \sum_{k=1}^{\infty}\left(1-\left|\varphi_{a}\left(a_{k}\right)\right|^{2}\right)<\infty .
$$


From Lemma 2.8, $\theta$ is a Carleson-Nemwman Blaschke product. Suppose that $f \in F(p, p-$ $2, s)$ and $f \theta \in B M O A$. Lemma 2.9 gives

$$
\sup _{z \in \Omega(\theta, \epsilon)}|f(z)|<\infty, \quad 0<\epsilon<1 .
$$

Thus,

$$
\begin{aligned}
& \sup _{a \in \mathbb{D}} \sum_{k=1}^{\infty}\left|f\left(a_{k}\right)\right|^{p}\left(1-\left|\varphi_{a}\left(a_{k}\right)\right|^{2}\right)^{s} \\
& \quad \leq\left(\sup _{z \in \Omega(\theta, \epsilon)}|f(z)|\right)^{p} \sup _{a \in \mathbb{D}} \sum_{k=1}^{\infty}\left(1-\left|\varphi_{a}\left(a_{k}\right)\right|^{2}\right)^{s}<\infty .
\end{aligned}
$$

Applying Lemma 2.6 implies that $f \theta \in F(p, p-2, s)$. Hence, $\theta$ is $(F(p, p-2, s), B M O A)$ improving.

(2) $\Rightarrow(1)$, (3) $\Rightarrow(2)$. Their proofs are obvious.

$(2) \Rightarrow(3)$. Let $\theta$ be $(F(p, p-2, s), B M O A)$-improving. If the inner function $\theta \in F(p, p-$ $2, s)$, then $\theta$ is a Carleson-Newman Blaschke product. For $f \in F(p, p-2, s)$, if $f \theta \in \mathcal{B}$, then any Carleson-Newman Blaschke product is $(B M O A, \mathcal{B})$-improving by Corollary 1 of [12]. Therefore, $f \theta \in B M O A$. Notice that $\theta$ is $(F(p, p-2, s), B M O A)$-improving, we have $f \theta \in F(p, p-2, s)$. Thus, $\theta$ is $(F(p, p-2, s), \mathcal{B})$-improving. The proof of Theorem 1 is completed.

\section{Proofs of Theorem 2 and Corollary 2}

In this section, we borrow the idea in [19] to study the zero set of $A B_{p}^{t}$ spaces. Following the proof of Lemma 2.6, we have the next result.

Lemma 4.1 Let $1<p<\infty, 0<t<1,0<t p<1$. Suppose $f \in A B_{p}^{t}, B$ is a Carleson-Newman Blaschke product with sequence of zeros $\left\{a_{k}\right\}_{k=1}^{\infty}$. Then $f B \in A B_{p}^{t}$ if and only if

$$
\sum_{k=1}^{\infty}\left|f\left(a_{k}\right)\right|^{p}\left(1-\left|a_{k}\right|^{2}\right)^{1-t p}<\infty
$$

When $p=q, \sigma=1$ or $\sigma=\frac{1}{p}$ in Theorem 1 of [13], we get the following lemma.

Lemma 4.2 Let $1 \leq p<\infty, 0<t<\frac{1}{p}, f \in H^{p}$. Then the following conditions are equivalent.

(1) $f \in A B_{p}^{t}$;

(2) $\int_{\mathbb{D}}\left(\int_{\partial \mathbb{D}}\left|f\left(e^{i \eta}\right)-f(z)\right| \frac{1-|z|^{2}}{\left|e^{i \eta}-z\right|} d \eta\right)^{p}\left(1-|z|^{2}\right)^{-t p-1} d A(z)<\infty$;

(3) $\int_{\mathbb{D}} \int_{\partial \mathbb{D}}\left|f\left(e^{i \eta}\right)-f(z)\right|^{p} \frac{1-|z|^{2}}{\left|e^{i \eta}-z\right|} d \eta\left(1-|z|^{2}\right)^{-t p-1} d A(z)<\infty$.

Proof of Theorem $2(1) \Rightarrow(2)$. Since $\left\{a_{k}\right\}_{k=1}^{\infty}$ is an $A B_{p}^{t}$-zero set, there exists $f \in A B_{p}^{t}$ such that $f\left(a_{k}\right)=0$ nowhere else. By $A B_{p}^{t} \subset H^{p}$, there exists a Blaschke product $B$, a singular inner function $S$ and an outer function $g$, such that $f=B S g$. Since $S \neq 0, g \neq 0, B$ is a Carleson-Newman Blaschke product with zeros $\left\{a_{k}\right\}_{k=1}^{\infty}$, from Corollary 3.1 of [29], we 
know that $A B_{p}^{s}$ has the $f$-property (see [30] for the definition of the $f$-property). Then

$$
g B=\frac{f}{S} \in A B_{p}^{t}
$$

Applying Lemma 4.1, we have (2).

$(2) \Rightarrow(1)$. It is obvious from Lemma 4.1.

$(3) \Rightarrow(2)$. By the Poisson integral formula

$$
g\left(a_{k}\right)=\frac{1}{2 \pi} \int_{\partial \mathbb{D}} g\left(e^{i \eta}\right) \frac{1-\left|a_{k}\right|^{2}}{\left|e^{i \eta}-a_{k}\right|^{2}} d \eta .
$$

Applying Hölder's inequality, we have

$$
\left|g\left(a_{k}\right)\right|^{p} \lesssim \int_{\partial \mathbb{D}}\left|g\left(e^{i \eta}\right)\right|^{p} \frac{1-\left|a_{k}\right|^{2}}{\left|e^{i \eta}-a_{k}\right|^{2}} d \eta .
$$

The desired result follows.

$(2) \Rightarrow(3)$. By the $p$-triangle inequality, we obtain

$$
\begin{aligned}
& \sum_{k=1}^{\infty}\left(1-\left|a_{k}\right|^{2}\right)^{2-t p} \int_{\partial \mathbb{D}} \frac{\left|g\left(e^{i \eta}\right)\right|^{p}}{\left|e^{i \eta}-a_{k}\right|^{2}} d \eta \\
& \quad \lesssim \sum_{k=1}^{\infty}\left(1-\left|a_{k}\right|^{2}\right)^{2-t p} \int_{\partial \mathbb{D}} \frac{\left|g\left(a_{k}\right)\right|^{p}+\left|g\left(e^{i \eta}\right)-g\left(a_{k}\right)\right|^{p}}{\left|e^{i \eta}-a_{k}\right|^{2}} d \eta .
\end{aligned}
$$

Let

$$
I_{1}=: \sum_{k=1}^{\infty}\left(1-\left|a_{k}\right|^{2}\right)^{2-t p} \int_{\partial \mathbb{D}} \frac{\left|g\left(a_{k}\right)\right|^{p}}{\left|e^{i \eta}-a_{k}\right|^{2}} d \eta
$$

and

$$
I_{2}=: \sum_{k=1}^{\infty}\left(1-\left|a_{k}\right|^{2}\right)^{2-t p} \int_{\partial \mathbb{D}} \frac{\left|g\left(e^{i \eta}\right)-g\left(a_{k}\right)\right|^{p}}{\left|e^{i \eta}-a_{k}\right|^{2}} d \eta
$$

It is obvious that

$$
\begin{aligned}
I_{1} & =\sum_{k=1}^{\infty}\left(1-\left|a_{k}\right|^{2}\right)^{1-t p}\left|g\left(a_{k}\right)\right|^{p} \int_{\partial \mathbb{D}} \frac{\left(1-\left|a_{k}\right|^{2}\right)}{\left|e^{i \eta}-a_{k}\right|^{2}} d \eta \\
& \asymp \sum_{k=1}^{\infty}\left(1-\left|a_{k}\right|^{2}\right)^{1-t p}\left|g\left(a_{k}\right)\right|^{p}<\infty .
\end{aligned}
$$

We next estimate $I_{2}$. Since $\left\{a_{k}\right\}_{k=1}^{\infty}$ is a finite union of interpolating sequences, we can write

$$
\left\{a_{k}\right\}_{k=1}^{\infty}=: \bigcup_{1 \leq i \leq n} \bigcup_{1 \leq j \leq \infty}\left\{a_{i j}\right\}
$$


and there exist a positive integer $n$ and $\delta_{i}>0, i=1,2, \ldots, n$, such that

$$
\prod_{j \neq l} \varrho\left(a_{i j}, a_{i l}\right) \geq \delta_{i}
$$

Then for fixed $i$, the pseudo-hyperbolic disks $\left\{E\left(a_{i j}, \frac{\delta_{i}}{4}\right)\right\}_{j=1}^{\infty}$ are pairwise disjoint. Notice that

$$
G(z)=: \int_{0}^{2 \pi}\left|g\left(e^{i \eta}\right)-g(z)\right|^{p} \frac{1-|z|^{2}}{\left|e^{i \eta}-z\right|^{2}} d \eta
$$

has the generalized sub-mean-value property. Therefore,

$$
\begin{aligned}
I_{2} & =\sum_{i=1}^{n} \sum_{j=1}^{\infty}\left(1-\left|a_{i j}\right|^{2}\right)^{1-t p} \int_{0}^{2 \pi}\left|g\left(e^{i \eta}\right)-g\left(a_{i j}\right)\right|^{p} \frac{1-\left|a_{i j}\right|^{2}}{\left|e^{i \eta}-a_{i j}\right|^{2}} d \eta \\
& \lesssim \sum_{i=1}^{n} \sum_{j=1}^{\infty}\left(1-\left|a_{i j}\right|^{2}\right)^{-1-t p} \int_{E\left(a_{i j}, \frac{\delta_{i}}{4}\right)} G(u) d A(u) \\
& \lesssim \sum_{i=1}^{n} \sum_{j=1}^{\infty} \int_{E\left(a_{i j}, \frac{\delta_{i}}{4}\right)} \frac{G(u)}{(1-|u|)^{1+t p}} d A(u) \\
& \lesssim \int_{\mathbb{D}} \frac{G(u)}{(1-|u|)^{1+t p}} d A(u) .
\end{aligned}
$$

Bearing in mind Lemma 4.2, we have (3).

Now, we prove that $G(z)$ has the generalized sub-mean-value property. Since $\mid g\left(e^{i \eta}\right)-$ $\left.g(z)\right|^{p}$ is subharmonic, using the sub-mean-value property, we have

$$
\left|g\left(e^{i \eta}\right)-g(z)\right|^{p} \lesssim \frac{1}{|E(z, \delta)|} \int_{E(z, \delta)}\left|g\left(e^{i \eta}\right)-g(w)\right|^{p} d A(w), \quad 0<\delta<1 .
$$

From [26, p.69 and Lemma 4.30], we know

$$
1-|z| \asymp 1-|w| \asymp|1-\bar{z} w|, \quad|1-\bar{a} w| \asymp|1-\bar{a} z|
$$

for $w \in E(z, \delta), 0<\delta<1, a \in \overline{\mathbb{D}}$. Thus, it follows that

$$
\begin{aligned}
G(z) & \lesssim \frac{1}{|E(z, \delta)|} \int_{E(z, \delta)} \int_{\partial \mathbb{D}}\left|g\left(e^{i \eta}\right)-g(w)\right|^{p} \frac{1-|w|^{2}}{\left|e^{i \eta}-w\right|^{2}} d \eta d A(w) \\
& =\frac{1}{|E(z, \delta)|} \int_{E(z, \delta)} G(w) d A(w) .
\end{aligned}
$$

That is, $G(z)$ has the generalized sub-mean-value property.

(2) $\Leftrightarrow(4)$. The proof is similar to that of (2) $\Leftrightarrow$ (3) and thus is omitted. The theorem is proved.

Lemma 4.3 Let $1 \leq p<\infty$ and $0<s<1$. Suppose $f=I_{f} \mathcal{O}_{f} \in F(p, p-2, s)$, where $I_{f}$ and $\mathcal{O}_{f}$ are inner-outer factors. Let I be an inner function dividing $I_{f}$. Then $f / I \in F(p, p-2, s)$. 
Proof Let $f \in F(p, p-2, s) \subseteq A B_{p}^{\frac{1-s}{p}}$. By the Möbius invariant property of $F(p, p-2, s)$, we have $f \circ \varphi_{a} \in F(p, p-2, s)$. From Corollary 3.1 of [29], we get

$$
\begin{aligned}
& \int_{\mathbb{D}}\left|\left(\frac{f(z)}{I(z)}\right)^{\prime}\right|^{p}\left(1-|z|^{2}\right)^{p-2}\left(1-\left|\varphi_{a}(z)\right|^{2}\right)^{s} d A(z) \\
& \quad=\int_{\mathbb{D}}\left|\left(\frac{f \circ \varphi_{a}(z)}{I \circ \varphi_{a}(z)}\right)^{\prime}\right|^{p}\left(1-|z|^{2}\right)^{p-2+s} d A(z) \\
& \quad \lesssim \int_{\mathbb{D}}\left|\left(f \circ \varphi_{a}\right)^{\prime}(z)\right|^{p}\left(1-|z|^{2}\right)^{p-2+s} d A(z) \\
& \quad=\int_{\mathbb{D}}\left|f^{\prime}(z)\right|^{p}\left(1-|z|^{2}\right)^{p-2}\left(1-\left|\varphi_{a}(z)\right|^{2}\right)^{s} d A(z) .
\end{aligned}
$$

The desired result follows.

Proof of Corollary 2 Taking the same sequence $\left\{a_{k}\right\}_{k=1}^{\infty}$ as in Proposition 1. Let $B(z)$ be the associated Blaschke product. Notice the fact that $F(p, p-2, t) \subseteq A B_{p}^{\frac{1-t}{p}}$, it implies that $\left\{a_{k}\right\}_{k=1}^{\infty}$ is not a $F(p, p-2, t)$-zero set. We deduce that $B(z)$ does not belong to $F(p, p-2, s)$. Combining this with Lemma 4.3, the proof of the rest is similar to that of Theorem 2 of [12] and thus is omitted.

\section{Competing interests}

The authors declare that they have no competing interests.

\section{Authors' contributions}

All authors contributed equally in the preparation of this article. All authors read and approved the final manuscript.

\section{Acknowledgements}

This work was supported by NSF of China (No. 11171203) and NSF of Guangdong Province (Grant No. 10151503101000025 and No. S2011010004511).

Received: 18 March 2014 Accepted: 16 July 2014 Published: 21 Aug 2014

\section{References}

1. Garnett, J: Bounded Analytic Functions. Academic Press, New York (1981)

2. Ahern, P: The mean modulus of derivative of an inner function. Indiana Univ. Math. J. 28, 311-347 (1979)

3. Ahern, P, Clark, DN: On inner functions with $H^{P}$ derivative. Mich. Math. J. 21, 115-127 (1974)

4. Ahern, P, Clark, DN: On inner functions with $B^{p}$ derivative. Mich. Math. J. 23, 107-118 (1976)

5. Gluchoff, A: On inner functions with derivative in Bergman spaces. III. J. Math. 31, 518-527 (1987)

6. Kim, HO: Derivatives of Blaschke products. Pac. J. Math. 114, 175-190 (1984)

7. Dyakonov, K: Self-improving behaviour of inner functions as multipliers. J. Funct. Anal. 240, 429-444 (2006)

8. Zhao, R: On a general family of function spaces. Ann. Acad. Sci. Fenn., Math. Diss. 105, 1-56 (1996)

9. Xiao, J: Holomorphic $\mathcal{Q}$ Classes. LNM, vol. 1767. Springer, Berlin (2001)

10. Xiao, J: Geometric $\mathcal{Q}_{p}$ Functions. Birkhäuser, Basel (2006)

11. Aulaskari, P, Lappan, P: Criteria for an analytic function to be Bloch and a harmonic or meromorphic function to be normal. In: Complex Analysis and Its Applications, Hong Kong, 1993. Pitman Res. Notes Math. Ser., vol. 305, pp. 136-146. Longman, Harlow (1994)

12. Peláez, J: Inner functions as improving multipliers. J. Funct. Anal. 255, 1403-1418 (2008)

13. Dyakonov, K: Besov spaces and outer functions. Mich. Math. J. 45, 143-157 (1998)

14. Carleson, L: On a class of meromorphic functions and its associated exceptional sets. Uppsala (1950)

15. Carleson, L: On the zeros of functions with bounded Dirichlet integrals. Math. Z. 56, 289-295 (1952)

16. Caughran, J: Two results concerning the zeros of functions with finite Dirichlet integral. Can. J. Math. 21, 312-316 (1969)

17. Shapiro, $H$, Shields, A: On the zeros of functions with finite Dirichlet integral and some related function spaces. Math. Z. 80, 217-229 (1962)

18. Taylor, B, Williams, D: Zeros of Lipschitz functions analytic in the unit disc. Mich. Math. J. 18, 129-139 (1971)

19. Pau, J, Peláez, J: On the zeros of functions in Dirichlet-type spaces. Trans. Am. Math. Soc. 363, 1981-2002 (2011)

20. Girela, D: Analytic functions of bounded mean oscillation. In: Complex Function Spaces (Mekrijärvi, 1999). Univ. Joensuu Dept. Math. Rep. Ser., vol. 4, pp. 61-170. Univ. Joensuu, Joensuu (2001) 
21. Arcozzi, N, Blasi, D, Pau, J: Interpolating sequences on analytic Besov type spaces. Indiana Univ. Math. J. 58, 1281-1318 (2009)

22. Pérez-González, F, Rättyä, J: Inner functions in the Möbius invariant Besov-type spaces. Proc. Edinb. Math. Soc. 52 , 751-770 (2009)

23. Ortega, JM, Fàbrega, J: Pointwise multipliers and Corona type decomposition in BMOA. Ann. Inst. Fourier (Grenoble) 46, 111-137 (1996)

24. Zhao, R: Distances from Bloch functions to some Möbius invariant spaces. Ann. Acad. Sci. Fenn., Math. Diss. 33, 303-313 (2008)

25. Blasi, D, Pau, J: A characterization of Besov type spaces and applications to Hankel type operators. Mich. Math. J. 56, 401-417 (2008)

26. Zhu, K: Operator Theory in Function Spaces. Am. Math. Soc., Providence (2007)

27. McDonald, G, Sundberg, C: Toeplitz operators on the disc. Indiana Univ. Math. J. 28, 595-611 (1979)

28. Dyakonov, K: Division and multiplication by inner functions and embedding theorems for star-invariant subspaces. Am. J. Math. 115, 881-902 (1993)

29. Böe, B: A norm on the holomorphic Besov space. Proc. Am. Math. Soc. 131, 235-241 (2003)

30. Hedenmalm, H: On the $f$ - and K-properties of certain function spaces. Contemp. Math. 91, 89-91 (1989)

10.1186/1029-242X-2014-312

Cite this article as: Lou and Qian: Inner functions as improving multipliers and zero sets of Besov-type spaces. Journal of Inequalities and Applications 2014, 2014:312

\section{Submit your manuscript to a SpringerOpen ${ }^{\ominus}$ journal and benefit from:}

- Convenient online submission

- Rigorous peer review

- Immediate publication on acceptance

- Open access: articles freely available online

- High visibility within the field

- Retaining the copyright to your article 